\title{
Evidence for IFNa-induced, SAMHD1-independent inhibitors of early HIV-1 infection
}

\author{
Caroline Goujon ${ }^{1 \dagger}$, Torsten Schaller ${ }^{1 \dagger}$, Rui Pedro Galão ${ }^{1}$, Sarah M Amie², Baek Kim², Kevin Olivieri ${ }^{3}$ \\ Stuart JD Neil ${ }^{1}$ and Michael $\mathrm{H}_{\text {Malim }}{ }^{{ }^{*}}$
}

\begin{abstract}
Background: Type I interferon (IFN) treatment of some cells, including dendritic cells, macrophages and monocytic THP-1 cells, restricts HIV-1 infection and prevents viral cDNA accumulation. Sterile alpha motif and HD domain protein 1 (SAMHD1), a dGTP-regulated deoxynucleotide triphosphohydrolase, reduces HIV-1 infectivity in myeloid cells, likely by limiting dNTPs available for reverse transcription, and has been described as IFNa-inducible. Myeloid cell infection by HIV-1 is enhanced by HIV-2/SIV $V_{S M}$ Vpx, which promotes SAMHD1 degradation, or by exogenous deoxyribonucleoside (dN) addition.
\end{abstract}

Findings: SAMHD1 expression was not substantially influenced by IFNa treatment of monocyte-derived macrophages or THP-1 cells. The contributions of SAMHD1 to the inhibition of HIV-1 infectivity by IFNa were assessed through the provision of Vpx, exogenous dN addition, or via RNAi-mediated SAMHD1 knock-down. Both $V p x$ and $d N$ efficiently restored infection in IFNa-treated macrophages, albeit not to the levels seen with these treatments in the absence of IFNa. Similarly using differentiated THP-1 cells, the addition of Vpx or dNs, or SAMHD1 knock-down, also stimulated infection, but failing to match the levels observed without IFNa. Neither Vpx addition nor SAMHD1 knock-down reversed the IFNa-induced blocks to HIV-1 infection seen in dividing U87-MG or THP-1 cells. Therefore, altered SAMHD1 expression or function cannot account for the IFNa-induced restriction to HIV-1 infection seen in many cells and cell lines.

Conclusion: IFNa establishes an anti-HIV-1 phenotype in many cell types, and appears to accomplish this without potentiating SAMHD1 function. We conclude that additional IFNa-induced suppressors of the early stages of HIV-1 infection await identification.

Keywords: HIV-1, Interferon, Restriction, Macrophages, SAMHD1, Vpx, Deoxyribonucleosides

\section{Findings}

Type I interferon (IFN) treatment of some cell types, including macrophages, dendritic cells and the monocytic cell line THP-1, potently induces a block to HIV-1 infection at the level of viral DNA accumulation [1-4]. The identities and roles of participating IFN-induced anti-HIV-1 host factors are yet to be defined. The HIV-2/SIV ${ }_{S M}$ Vpx protein greatly increases the permissivity of myeloid cells to HIV-1 infection [5,6]. The protein sterile alpha motif (SAM) histidine/aspartic acid (HD) domain containing 1 (SAMHD1) was recently identified as a target for Vpx-

\footnotetext{
* Correspondence: michael.malim@kcl.ac.uk

'Equal contributors

'Department of Infectious Diseases, King's College London, 2nd Floor Borough Wing, Guy's Hospital, London Bridge, London SE1 9RT, UK Full list of author information is available at the end of the article
}

induced proteasomal degradation in monocyte-derived macrophages (MDMs) and dendritic cells, as well as in quiescent CD4 T-cells [7-10]. SAMHD1 is a dGTPregulated deoxynucleotide triphosphohydrolase that limits the pool of dNTPs available for reverse transcription, therefore reducing HIV-1 infection of myeloid cells [11,12]; for a short review see [13]. Interestingly, SAMHD1 has been reported to be IFN $\gamma$ - and IFN $\alpha$-inducible in human dendritic cells and monocytes, respectively $[14,15]$. And consistent with this, it was also shown that Vpx enhances HIV-1 infection of IFN $\alpha$-treated monocyte-derived dendritic cells [16]. In this context, we sought to investigate further the role of SAMHD1 in IFN $\alpha$-induced HIV-1 restriction.

To address the effects of type I IFN on SAMHD1 expression, we examined a variety of primary cell types and

\section{Biomed Central}


immortalized cell lines. MDMs and activated $\mathrm{CD}^{+} \mathrm{T}$ cells, both obtained from multiple donors, dividing and PMA-treated (differentiated) THP-1 and U937 cells, as well as U87-MG glioblastoma cells, were treated with $1000 \mathrm{U} / \mathrm{ml} \mathrm{IFN \alpha}$ for $24 \mathrm{~h}$ (for detailed description of experimental procedures, see Additional file 1). The cells were harvested for RNA extraction and RT-qPCR, or for western blot analysis (Figure 1). In MDMs, $\mathrm{CD} 4^{+} \mathrm{T}$ cells and dividing THP-1 cells, SAMHD1 was poorly IFN $\alpha-$ inducible $(<2$-fold increase in RNA abundance, Figure 1A; no difference was observed at the protein level for MDMs, Figure 1B), in contrast to two well-known IFN $\alpha$ stimulated genes (ISGs), ISG15 and APOBEC3A [17-20]. In PMA-treated THP-1, untreated U87-MG, as well as in PMA-treated or dividing U937 cells, SAMHD1 mRNA levels were modestly upregulated by IFN $\alpha$ treatment (3- to 5 -fold), possibly suggesting a cell type dependent effect of IFN $\alpha$ on SAMHD1 expression. Of note, U937 cells expressed more than one order of magnitude lower levels of SAMHD1 compared to MDMs or THP-1 cells, however IFN $\alpha$ treatment only increased SAMHD1 RNA levels by 3 - to 5 -fold. The IFNo-induced block to HIV-1 infection is observed in macrophages, PMA-treated and dividing THP-1 cells as well as in U87-MG cells, but not in U937 cells ([1]; and this manuscript), suggesting that induction of SAMHD1 expression by IFN $\alpha$ does not directly correlate with a block to HIV-1 infection. However, since SAMHD1 activity rather than abundance may be regulated by IFN $\alpha$, we further investigated a possible role for SAMHD1 in the IFN $\alpha$-induced suppression of HIV-1.

As both Vpx-containing virus-like particles (Vpx-VLPs) as well as exogenous deoxyribonucleosides $(\mathrm{dN})$ relieve the SAMHD1-mediated HIV-1 block in myeloid cells $[7,8,12]$, we evaluated the effects of these conditions on IFNo-treated MDMs (Figure 2). MDMs from 4 independent donors were treated or not with IFNa for $24 \mathrm{~h}$ prior to infection, and incubated with $\mathrm{dN}(0.5$ or $2.5 \mathrm{mM})$, or with Vpx-VLPs, or left untreated (control). The cells were then infected with increasing amounts of a VSV-G pseudotyped HIV-1 encoding GFP reporter virus. The percentage of infected MDMs was enumerated by flow cytometry 2 days later (Figure 2A displays the mean data and Additional file 2: Figure S1A separates the data by donor). In agreement with previous reports $[5,12,21]$, both Vpx-VLPs and exogenous dN increased HIV-1 infectivity in MDMs in the absence of IFN (6- to 9-fold and 3- to 8-fold, respectively; Figure 2A). As we reported previously, IFN $\alpha$ treatment decreased HIV-1 infectivity in MDMs by at least 35 -fold [1]. Strikingly, both Vpx and $2.5 \mathrm{mM}$ dN efficiently stimulated HIV-1 infection in IFN $\alpha$-treated MDMs (by more than 100-fold at the highest MOI). However, the levels of infection did not reach the levels observed without IFN $\alpha$ for either condition. Importantly, control VLPs that lacked Vpx did not have any positive impact on the

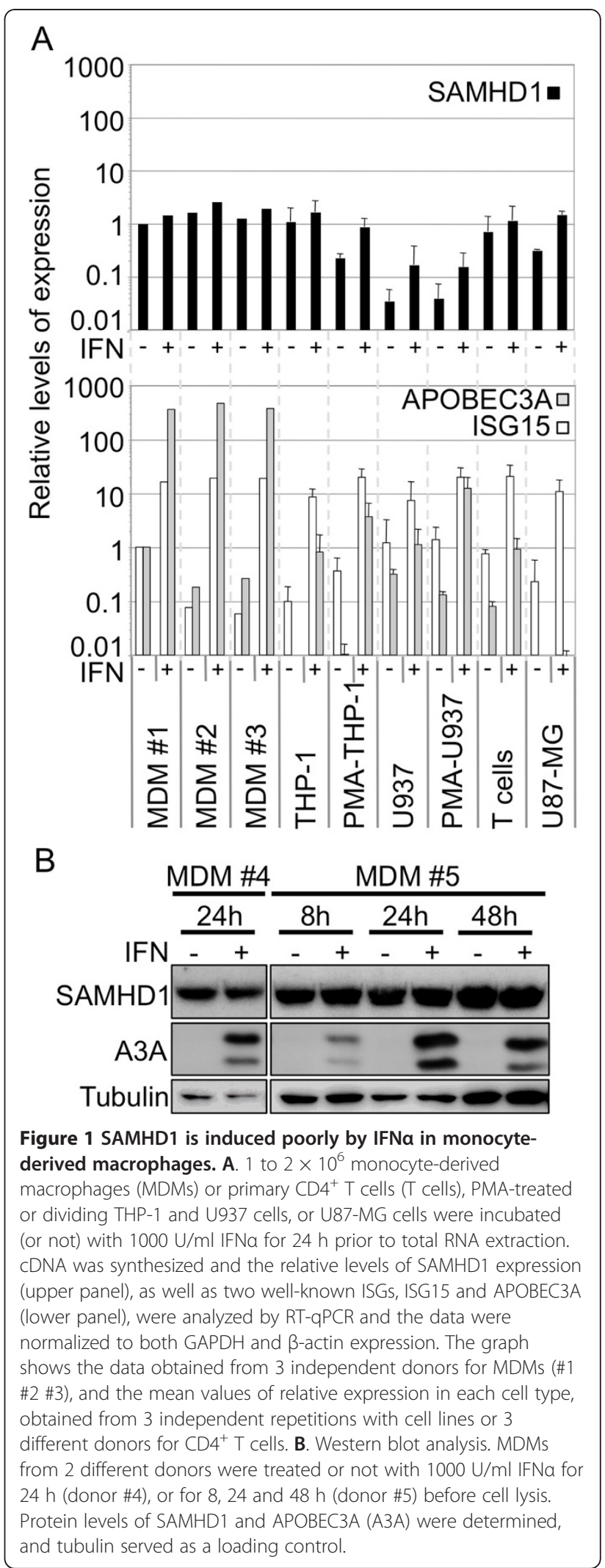




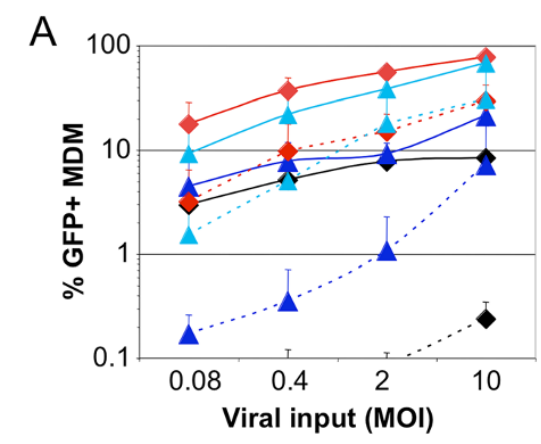

B

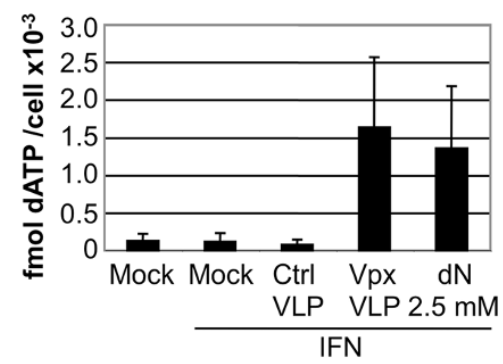

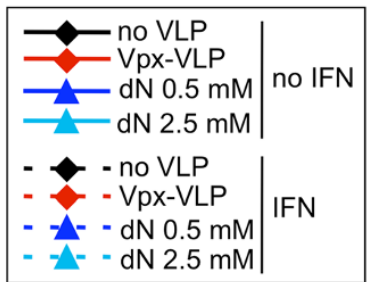

C

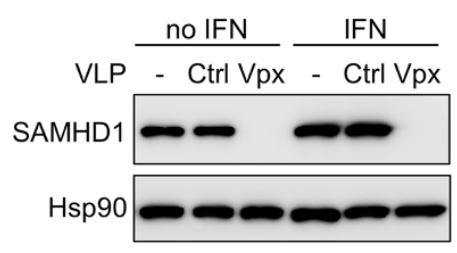

Figure 2 Vpx-VLPs or exogenous dN treatment greatly enhance HIV-1 infection in IFNa-treated MDMs. A. VSV-G pseudotyped HIV-1 derived GFP reporter virus was produced in 293T cells and used to infect control or IFNa-treated MDMs at different MOls (0.08 to 10 , as determined in 293T cells), in the presence or the absence of either Vpx-VLP or $0.5 \mathrm{mM}$ or $2.5 \mathrm{mM}$ deoxyribonucleosides (dN). Levels of infection were monitored using flow cytometry to measure the percentage of cells expressing GFP, and mean values from 4 independent experiments (using cells from 4 different donors) are shown. Notably, treatment of MDMs with the higher concentration of dN changed their morphology, with the cells becoming rounder and less adherent. We conclude that experiments using high dN doses must be analysed with caution. B MDMs were treated or not with IFNa for $24 \mathrm{~h}$ and subsequently incubated or not with Ctrl-VLPS, Vpx-VLPs or dN (at 0.5 or 2.5 mM as indicated) for $16 \mathrm{~h}$ before lysis. Whole cell dATP was quantified using a single nucleotide quantification assay. Mean values from 4 independent experiments (using cells from 4 different donors) are shown. C. Western blot analysis. Cell lysates from MDMs (donor \#4) were harvested 24 h post IFNa and Vpx-VLP treatment, and SAMHD1 expression was analysed by western blot; Hsp90 served as a loading control.

efficiency of infection either in the absence or presence of IFNa (Additional file 2: Figure S1A).

The positive effect of both $\mathrm{Vpx}$ and $\mathrm{dN}$ correlated with significant increases in the intracellular dNTP pool (as shown by intracellular dATP measurement, Figure 2B displays the mean data and Additional file 2: Figure S1B shows the results for each donor). In agreement with this finding, $\mathrm{Vpx}$ efficiently induced the degradation of SAMHD1 (Figure 2C). Closer inspection of these data reveals that $\mathrm{dN}$ provision or $\mathrm{Vpx}$ addition promote infection to greater magnitudes in cultures treated with IFN $\alpha$. While the molecular basis for this is not yet known, it is plausible that, although its expression is not increased, SAMHD1 activity might be potentiated in IFN $\alpha$-treated MDMs. However, measurement of dATP levels in the presence or absence of IFN $\alpha$ did not reveal a significant difference in dATP concentrations, implying that the dNTP hydrolase activity of SAMHD1 is not measurably affected by IFN $\alpha$ treatment (Figure $2 \mathrm{~B}$ and Additional file 2: Figure S1B). Alternatively, other restriction factor(s) might suppress HIV-1 infection more effectively when cellular dNTP concentrations are low. Of note, MDMs from different donors behave differently with respect to the rescue of $\mathrm{HIV}-1$ infectivity following $\mathrm{dN}$ treatment, suggesting that donor-specific differences in SAMHD1 levels or activity contribute to differences in rescue by $\mathrm{dN}$ or $\mathrm{Vpx}$ (see donors 1 and 2, versus donors 3 and 4 in Additional file 2: Figure S1).

We next evaluated the effects of $\mathrm{dN}$ and $\mathrm{Vpx}$ treatment on IFN $\alpha$-treated THP-1 cells (Figure 3). PMA-differentiated THP-1 cells (Figure 3A) or dividing THP-1 cells (Figure 3B) were treated or not with IFN $\alpha$ for $24 \mathrm{~h}$, and incubated with $\mathrm{dN}(0.5 \mathrm{mM})$, or with Vpx-VLPs, or left untreated (control) before infection with increasing amounts of a VSV-G pseudotyped HIV-1 encoding GFP reporter virus. In agreement with previous reports $[12,21]$, both Vpx-VLPs and exogenous $\mathrm{dN}$ increased HIV-1 infectivity in PMA-THP-1 cells in the absence of IFN $\alpha(\sim 2$-fold; Figure 3A), but had no significant effect on HIV-1 infection of dividing THP-1 cells (Figure 3B). As reported previously, IFN $\alpha$ treatment decreased HIV-1 infectivity in THP-1 cells by at least 10 -fold [1]. In PMA-treated THP-1 cells, both treatments partially increased HIV-1 infectivity following IFN $\alpha$ treatment, but had no significant effect in dividing THP-1 cells. Western blot analysis showed 

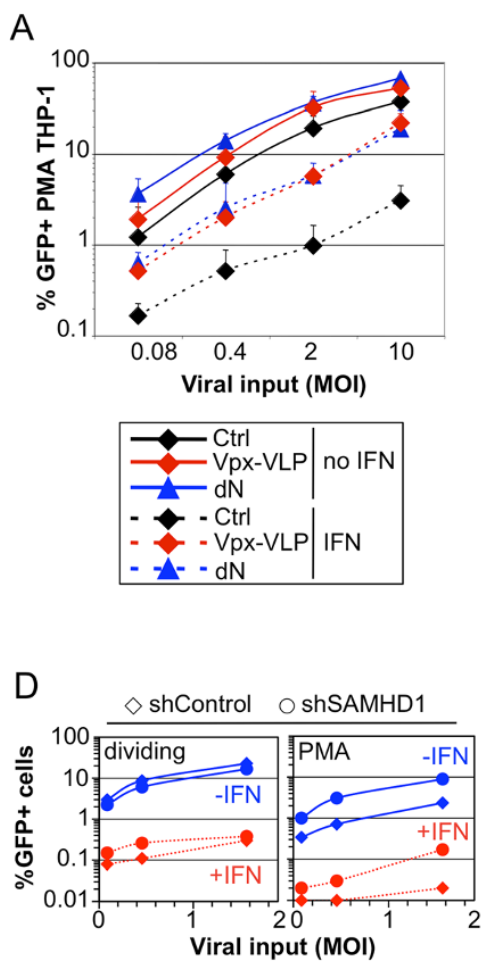

B
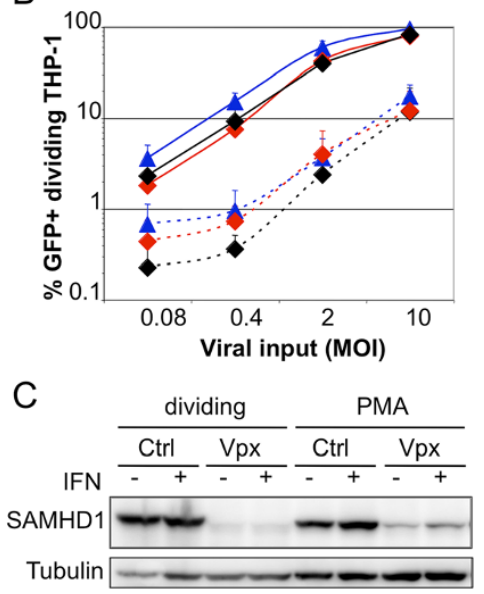

$\mathrm{E}$

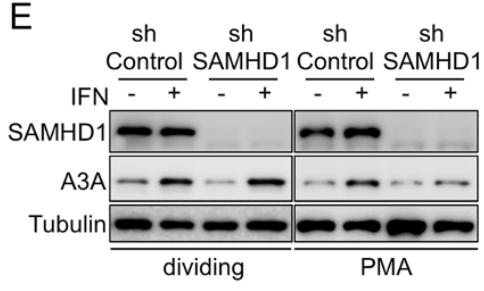

Figure 3 Vpx-VLPs, exogenous dN treatment or SAMHD1 knock-down do not rescue the IFNa-induced block to HIV-1 infection in THP1 cells. A. and B. VSV-G pseudotyped HIV-1 derived GFP reporter virus was produced in 293T cells and used to infect control or IFNa-treated PMA-differentiated THP-1 cells (A), or dividing THP-1 cells (B) at different MOls (0.08 to 10), in the presence or the absence of either Vpx-VLP or $0.5 \mathrm{mM}$ deoxyribonucleosides (dN). Levels of infection were monitored using flow cytometry to measure the percentage of cells expressing GFP, and mean values from 3 independent experiments are shown. C. Western blot analysis. Cell lysates from THP-1 cells were harvested $24 \mathrm{~h}$ post IFNa and Vpx-VLP treatment, and SAMHD1 expression was analysed by western blot; tubulin served as a loading control. D. THP-1 cells expressing a control shRNA or shRNA targeting SAMHD1 were differentiated with PMA or not in the presence or absence of IFNa for $24 \mathrm{~h}$. Cells were infected with 3 different doses of VSV-G pseudotyped GFP reporter virus for $48 \mathrm{~h}$, and GFP positive cells were enumerated by flow cytometry. The data are representative of 3 independent experiments with two independent shRNAs against SAMHD1. E. Western blot analysis of parallel samples from D. Protein levels of SAMHD1 and APOBEC3A (A3A) were determined and tubulin served as a loading control.

that Vpx-VLPs induced the degradation of SAMHD1 under all conditions (Figure 3C), indicating that IFN $\alpha$ mediated suppression of HIV-1 infection in dividing and non-dividing THP-1 cells is maintained to significant degrees following SAMHD1 removal.

To investigate further the relationship between IFN $\alpha$ action and SAMHD1 function, we evaluated the effect of IFN $\alpha$ in THP-1 cells selectively depleted of SAMHD1 under dividing and differentiated culture conditions (Figure 3D). THP-1 cells were transduced with an HIV-1 shRNA vector targeting SAMHD1, or a control, and selected with puromycin. Dividing or PMA-treated shControl or shSAMHD1 THP-1 cells were treated with IFN $\alpha$ or not for $24 \mathrm{~h}$, and then infected with different doses of VSV-G pseudotyped HIV-1 GFP vector (Figure 3D). As previously reported, reducing SAMHD1 protein levels increased the permissivity of PMA-treated (non-dividing) THP-1 cells by $\sim 4$-fold (Figure 3D) $[7,15]$. IFN $\alpha$ treatment decreased HIV-1 infectivity by more than one order of magnitude, irrespective of whether SAMHD1 was present or not, or whether the cells were dividing (Figure $3 \mathrm{~A}$ and $\mathrm{B}$ ). Although it is possible that low residual amounts of SAMHD1 were able to exert an effect, the data collectively suggest that SAMHD1 is, if at all, only a minor contributor to the IFN $\alpha$-induced early block to HIV-1 infection in these cells. Of note, similar results were obtained in challenges with VSV-G pseudotyped full length HIV-1 (not shown).

SAMHD1 is highly expressed in MDMs and THP-1 cells, but to a lower extent in U87-MG cells and we found that IFN $\alpha$ treatment increased SAMHD1 expression in U87-MG cells to levels close to those observed in THP-1 or MDMs (Figure 1A) and western blot analysis confirmed increased protein levels (Figure 4B). To test for a cell-type specific activity of SAMHD1 after IFN $\alpha$ treatment we investigated whether Vpx-VLP treatment would rescue HIV-1 infectivity in IFN $\alpha$-treated U87-MG cells. Cultures were treated or not for $24 \mathrm{~h}$ with IFNo, incubated either with Vpx-VLPs, with VLPs devoid of Vpx (Ctrl-VLPs) or left untreated, and then 

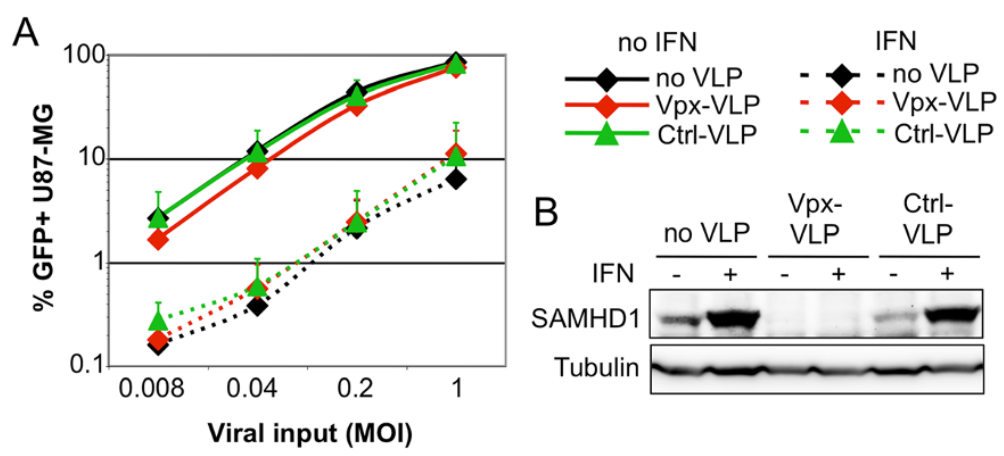

Figure 4 SAMHD1 is induced by IFNa in U87-MG cells, but Vpx triggered degradation does not rescue IFNa-mediated HIV-1

suppression. A. U87-MG cells were treated for $24 \mathrm{~h}$ with IFNa, or not, and incubated with no VLPS, Vpx-VLPS, or control VLPS (Ctrl-VLPS) and then infected with increasing amounts of GFP reporter virus (MOI 0.008 to 1), and GFP expression monitored as before. The mean values from 3 independent experiments are shown. B. Western blot analysis of a parallel samples from A. Protein levels of SAMHD1 were determined and tubulin served as a loading control.

infected with increasing amounts of HIV-1 GFP vector (Figure 4A). As in myeloid cells (U937 cells excluded), IFN $\alpha$ treatment decreased HIV-1 infectivity in U87-MG cells by $\sim 10$-fold. Though SAMHD1 was efficiently degraded by Vpx-VLPs both in the absence or presence of IFN $\alpha$ (Figure 4B), the effects on HIV-1 infectivity were minor (maximum 2-fold increase, seen also with Ctrl-VLPs). In keeping with observations made in dividing THP-1 cells, this further demonstrates that SAMHD1 degradation does not necessarily correlate with a rescue of HIV-1 infection, and suggests that factors distinct from SAMHD1 are involved in the IFN $\alpha$-induced block to HIV-1 infection in dividing U87MG and THP-1 cells.

In this report, we used several primary and immortalized cell types to examine the relationship between SAMHD1 expression and function, and the inhibition of HIV-1 infection by IFNa. Taken together, our data reveal that SAMHD1, while previously established as an important regulator of HIV-1 infection [7-10], is not a major effector of IFN $\alpha$-mediated HIV-1 restriction in a number of cell types and lines. In particular, by taking advantage of the ability of Vpx to degrade SAMHD1 [7,8], dN addition to bypass its deoxynucleoside triphosphohydrolase activity, or selective SAMHD1 knock-down, our findings indicate that IFN is often able to suppress HIV-1 infectivity independently of SAMHD1 activity. In keeping with this, several of our experiments also showed that HIV-1 inhibitory phenotypes that bear the hallmarks of SAMHD1-mediated suppression are displayed in differentiated cultures of primary myeloid cells in the presence or absence of IFN $\alpha$. Interestingly, $\mathrm{Vpx}$ and $\mathrm{dN}$ provoked a substantial increase in infection of IFN $\alpha$-treated MDMs, which was greater than observed in untreated MDMs. This implies that either SAMHD1 function is at least partly responsible for the IFN $\alpha$-mediated restriction in this cell type and/or its ability to reduce the pool of dNTPs is necessary for the action of other restriction factor(s). For example, we speculate that SAMHD1 may allow viral RNA or RNA/DNA reverse transcription intermediates to be specifically inhibited by IFNo-induced restriction factors, whilst the presence of $\mathrm{Vpx}$ or $\mathrm{dN}$ addition results in these structures being less abundant (or protected) owing to efficient reverse transcription. Lastly, it has been suggested that $\mathrm{Vpx}$ binds to APOBEC3A (A3A) possibly contributing to increased HIV-1 infectivity in monocytic cells [22,23]. Although we cannot rule out a role for A3A in the IFN $\alpha$-induced block in MDMs described here, the rescue of HIV-1 infectivity by $\mathrm{dN}$ treatment argues against such a direct role. Moreover, A3A is not induced in U87-MG cells treated with IFN $\alpha$, despite a potent block to HIV-1 infection. Our studies in U87-MG and THP-1 cells, where manipulation of SAMHD1 fails to overcome the effects of IFN $\alpha$, allude to the existence of additional, possibly cell type specific, IFN $\alpha$-inducible (and Vpx-independent) inhibitors of the early stages of HIV-1 infection. Validation of these models will require the identification of such cell-encoded factors.

\section{Additional files}

Additional file 1: Material and methods.

Additional file 2: Figure S1. Vpx-VLPs or exogenous $d N$ treatment greatly improve HIV-1 infection in IFNa-treated MDMs. A. VSV-G pseudotyped HIV-1 derived GFP reporter virus was produced in 293T cells and used to infect control or IFNa-treated MDMs (from four different donors, donor \#1 to \#4) at different MOls (0.08 to 10), in the presence or the absence of either Vpx-VLP or $0.5 \mathrm{mM}$ or $2.5 \mathrm{mM}$ deoxyribonucleosides ( $\mathrm{dN}$ ). Levels of infection were monitored using flow cytometry to measure the percentage of MDMs expressing GFP. B. Single nucleotide-incorporation analysis of dATP from MDMs treated or not with IFNa for $24 \mathrm{~h}$ and subsequently incubated or not with Ctrl-VLPs, Vpx-VLPs or dN (at 0.5 or 2.5 mM) for 16 h before lysis (+ Ctrl: + dATP; - Ctrl: no dATP).

\section{Abbreviations}

Ctrl: Control; dATP: Deoxyadenosine triphosphate; dGTP: Deoxyguanosine triphosphate; dN: Deoxyribonucleoside; dNTP: Deoxyribonucleoside triphosphate; HIV-1: Human immunodeficiency virus type 1; IFNa: Interferon 
alpha; MDMs: Monocyte-derived macrophages; MOI: Multiplicity of infection; PMA: Phorbol-12-myristate-13-acetate; RNA: Ribonucleic acid; RNAi: RNA interference; SAMHD1: Sterile alpha motif (SAM) histidine/aspartic acid (HD) domain containing 1; shRNA: Short hairpin RNA; SIV: Simian immunodeficiency virus; VLP: Virus like particles; Vpx: Viral protein X.

\section{Competing interests}

The authors declare that they have no competing interests.

\section{Authors' contributions}

CG and TS designed the study, carried out the experiments and drafted the manuscript. RPG and SN helped design the study and provided reagents. SMA and BK did the AATP quantification. KO provided reagents. MHM designed the study and wrote the manuscript. All authors read and approved the final manuscript.

\section{Acknowledgements}

This work was supported by the U.K. Medical Research Council, the National Institutes of Health (Al049781, Al070072, Al077401, Al090935, DA033773), the European Community's Seventh Framework Programme [FP7/2007-2013] under grant agreement n'PIEF-GA-2009-237501 (to CG), and the Department of Health via a National Institute for Health Research comprehensive Biomedical Research Centre award to Guy's and St. Thomas' NHS Foundation Trust in partnership with King's College London and King's College Hospital NHS Foundation Trust.

\section{Author details}

'Department of Infectious Diseases, King's College London, 2nd Floor, Borough Wing, Guy's Hospital, London Bridge, London SE1 9RT, UK. ${ }^{2}$ Department of Microbiology and Immunology, University of Rochester Medical, Rochester, USA. ${ }^{3}$ Infectious and Inflammatory Disease Center, Sanford-Burnham Medical Research Institute, La Jolla, USA.

Received: 26 November 2012 Accepted: 19 February 2013 Published: 25 February 2013

\section{References}

1. Goujon C, Malim MH: Characterization of the alpha interferon-induced postentry block to HIV-1 infection in primary human macrophages and T cells. J Virol 2010, 84:9254-9266.

2. Cheney KM, McKnight A: Interferon-alpha mediates restriction of human immunodeficiency virus type-1 replication in primary human macrophages at an early stage of replication. PLoS One 2010, 5:e13521.

3. Baca-Regen L, Heinzinger N, Stevenson M, Gendelman HE: Alpha interferon-induced antiretroviral activities: restriction of viral nucleic acid synthesis and progeny virion production in human immunodeficiency virus type 1-infected monocytes. J Virol 1994, 68:7559-7565.

4. Meylan PR, Guatelli JC, Munis JR, Richman DD, Kornbluth RS: Mechanisms for the inhibition of HIV replication by interferons-alpha, -beta, and -gamma in primary human macrophages. Virology 1993, 193:138-148.

5. Goujon C, Jarrosson-Wuilleme L, Bernaud J, Rigal D, Darlix JL, Cimarelli A: With a little help from a friend: increasing HIV transduction of monocyte-derived dendritic cells with virion-like particles of SIV(MAC). Gene Ther 2006, 13:991-994.

6. Goujon C, Riviere L, Jarrosson-Wuilleme L, Bernaud J, Rigal D, Darlix JL, Cimarelli A: SIVSM/HIV-2 Vpx proteins promote retroviral escape from a proteasome-dependent restriction pathway present in human dendritic cells. Retrovirology 2007, 4:2.

7. Laguette N, Sobhian B, Casartelli N, Ringeard M, Chable-Bessia C, Segeral E, Yatim A, Emiliani S, Schwartz O, Benkirane M: SAMHD1 is the dendriticand myeloid-cell-specific HIV-1 restriction factor counteracted by Vpx. Nature 2011, 474:654-657.

8. Hrecka K, Hao C, Gierszewska M, Swanson SK, Kesik-Brodacka M, Srivastava S, Florens L, Washburn MP, Skowronski J: Vpx relieves inhibition of HIV-1 infection of macrophages mediated by the SAMHD1 protein. Nature 2011, 474:658-661.

9. Baldauf HM, Pan X, Erikson E, Schmidt S, Daddacha W, Burggraf M, Schenkova K, Ambiel I, Wabnitz G, Gramberg T, et al: SAMHD1 restricts HIV1 infection in resting CD4(+) T cells. Nat Med 2012, 18:1682-1687.
10. Descours B, Cribier A, Chable-Bessia C, Ayinde D, Rice G, Crow Y, Yatim A, Schawartz O, Laguette N, Benkirane M: SAMHD1 restricts HIV-1 reverse transcription in quiescent CD4+ T-cells. Retrovirology 2012, 9:87.

11. Goldstone DC, Ennis-Adeniran V, Hedden JJ, Groom HC, Rice Gl, Christodoulou E, Walker PA, Kelly G, Haire LF, Yap MW, et al: HIV-1 restriction factor SAMHD1 is a deoxynucleoside triphosphate triphosphohydrolase. Nature 2011, 480:379-382.

12. Lahouassa H, Daddacha W, Hofmann H, Ayinde D, Logue EC, Dragin L, Bloch N, Maudet C, Bertrand M, Gramberg T, et al: SAMHD1 restricts the replication of human immunodeficiency virus type 1 by depleting the intracellular pool of deoxynucleoside triphosphates. Nat Immunol 2011, 13:223-228.

13. Schaller T, Goujon C, Malim MH: AIDS/HIV. HIV interplay with SAMHD1. Science 2012, 335:1313-1314.

14. Li N, Zhang W, Cao X: Identification of human homologue of mouse IFNgamma induced protein from human dendritic cells. Immunol Lett 2000, 74:221-224.

15. Berger A, Sommer AF, Zwarg J, Hamdorf M, Welzel K, Esly N, Panitz S, Reuter A, Ramos I, Jatiani A, et al: SAMHD1-deficient CD14+ cells from individuals with Aicardi-Goutieres syndrome are highly susceptible to HIV-1 infection. PLoS Pathog 2011, 7:e1002425.

16. Pertel T, Reinhard C, Luban J: Vpx rescues HIV-1 transduction of dendritic cells from the antiviral state established by type 1 interferon. Retrovirology 2011, 8:49.

17. Sadler AJ, Williams BR: Interferon-inducible antiviral effectors. Nat Rev Immunol 2008, 8:559-568.

18. Peng G, Lei KJ, Jin W, Greenwell-Wild T, Wahl SM: Induction of APOBEC3 family proteins, a defensive maneuver underlying interferon-induced anti-HIV-1 activity. J Exp Med 2006, 203:41-46.

19. Koning FA, Newman EN, Kim EY, Kunstman KJ, Wolinsky SM, Malim MH: Defining APOBEC3 expression patterns in human tissues and hematopoietic cell subsets. J Virol 2009, 83:9474-9485.

20. Narvaiza I, Linfesty DC, Greener BN, Hakata Y, Pintel DJ, Logue E, Landau NR, Weitzman MD: Deaminase-independent inhibition of parvoviruses by the APOBEC3A cytidine deaminase. PLoS Pathog 2009, 5:e1000439.

21. Goujon C, Arfi V, Pertel T, Luban J, Lienard J, Rigal D, Darlix JL, Cimarelli A: Characterization of simian immunodeficiency virus SIVSM/human immunodeficiency virus type $2 \mathrm{Vpx}$ function in human myeloid cells. J Virol 2008, 82:12335-12345.

22. Berger A, Munk C, Schweizer M, Cichutek K, Schule S, Flory E: Interaction of VPX and APOBEC $3 A$ correlates with efficient lentivirus infection of monocytes. J Biol Chem 2010.

23. Berger G, Durand S, Fargier G, Nguyen XN, Cordeil S, Bouaziz S, Muriaux D, Darlix $J L$, Cimarelli $A$ : APOBEC3A is a specific inhibitor of the early phases of HIV-1 infection in myeloid cells. PLOS Pathog 2011, 7:e1002221.

doi:10.1186/1742-4690-10-23

Cite this article as: Goujon et al: Evidence for IFNa-induced, SAMHD1independent inhibitors of early HIV-1 infection. Retrovirology 2013 10:23.

\section{Submit your next manuscript to BioMed Central and take full advantage of:}

- Convenient online submission

- Thorough peer review

- No space constraints or color figure charges

- Immediate publication on acceptance

- Inclusion in PubMed, CAS, Scopus and Google Scholar

- Research which is freely available for redistribution 